\title{
SEMIGROUP PRODUCT FORMULAS AND ADDITION OF UNBOUNDED OPERATORS
}

\author{
BY PAUL R. CHERNOFF ${ }^{1}$ \\ Communicated by Paul Halmos, August 25, 1969
}

1. Introduction. Let $A$ and $B$ be selfadjoint operators on a Hilbert space. If these operators are unbounded the problem of interpreting their sum as a selfadjoint operator is not trivial. Of course, if the algebraic sum $A+B$, defined on the common domain $D(A) \cap D(B)$, is selfadjoint or has a selfadjoint closure there is no difficulty. But in general $A+B$ may have infinitely many different selfadjoint extensions; indeed, $D(A+B)$ may reduce to (0); or, on the other hand, $A+B$ need admit no selfadjoint extension.

One reason for the study of such questions arises in physics: in many quantum mechanical systems the Hamiltonian operator is the formal sum of a well-defined "free" Hamiltonian A and an "interaction" Hamiltonian B. It follows from a result of Trotter [5] that if the closure $\operatorname{cl}(A+B)=C$ is selfadjoint then the Lie product formula

$$
\lim _{n \rightarrow \infty}\left(e^{i t / n A} e^{i t / n B}\right)^{n}=e^{i t C}
$$

is valid. (For a proof of a more general product formula, see [1].) Interestingly, however, Nelson [4] demonstrated that the limit in (1) can exist even if $A+B$ is not essentially selfadjoint, and can successfully be used to define the dynamical group, and therefore the Hamiltonian operator, for certain quantum systems.

It is therefore of interest to study the general properties of product limits like the above, and in particular their use to define a generalized addition for unbounded operators. In this paper we shall state a number of results in this area. Proofs of these and additional results will be given elsewhere.

2. Product limits. Let $X$ be a Banach space. Let $F(t), 0 \leqq t<\infty$, be a strongly continuous function whose values are linear contraction

AMS Subject Classifications. Primary 4748, 4750; Secondary 8147.

Key Words and Phrases. Semigroups of operators, product formulas, unbounded operators, generalized addition, selfadjoint operators, Friedrichs extensions, Hamiltonian operators.

1 The results announced here are contained in the author's Ph.D. thesis, written under the direction of Professor G. W. Mackey at Harvard University. The author was partially supported by a National Science Foundation Graduate Fellowship. 
operators on $X$. We also require that $F(0)=I$, the identity. An example is given by $F(t)=P_{t} Q_{t}$ where $P_{t}$ and $Q_{t}$ are $\left(C_{0}\right)$ contraction semigroups on $X$. By the strong derivative $F^{\prime}(0)$ we mean the operator

$$
F^{\prime}(0) x=\lim _{t \rightarrow 0}(F(t) x-x) / t
$$

defined for all $x \in X$ for which the limit exists.

Theorem 2.1. Suppose that $F^{\prime}(0)$ is densely defined. Assume that for all $t \geqq 0$ the limit

$$
\lim _{n \rightarrow \infty} F(t / n)^{n}=R_{t}
$$

exists in the strong operator topology. Then $R_{t}$ is a $\left(C_{0}\right)$ contraction semigroup. The infinitesimal generator of $R_{t}$ is an extension of $F^{\prime}(0)$.

REMARK. Examples exist which show that the limit in (2) can exist with $F^{\prime}(0)$ not densely defined.

THEOREM 2.2. With no hypothesis on $F^{\prime}(0)$, suppose only that the limit in (2) exists in the strong topology. Then $R_{t}$ is a semigroup and is strongly continuous for $t>0$. (However, $R_{t}$ need not be continuous at 0 .)

Concerning the proofs of these theorems, it is easy to see that $R_{t}$ satisfies the functional equation

$$
R_{n t}=\left(R_{t}\right)^{n}
$$

for every positive integer $n$. It follows that $R_{s+t}=R_{s} R_{t}$ if the ratio $s / t$ is a rational number. The essential difficulty, therefore, is establishing the continuity of $R_{t}$. A special argument does this in 2.1. In the proof of the more general result 2.2 one makes use of the following proposition, which may be of some intrinsic interest.

Proposition 2.2.1. Let $R_{t}$ be a strongly measurable operator valued solution of the functional equation (3). Then there exists a semigroup $\widetilde{R}_{t}$, strongly continuous for $t>0$, such that $R_{t}=\widetilde{R}_{t}$ for a.e.t. $\widetilde{R}_{t}$ is a $\left(C_{0}\right)$ semigroup if and only if the essential span of the ranges of the operators $R_{t}$ is dense.

One can deduce that $R_{t} \equiv \widetilde{R}_{t}$ if one knows that $R_{t}$ has a "large" set of points of continuity in the sense of Baire category, and this is in fact the case under the hypotheses of 2.2.

Corollary 2.3. If $H$ is a Hilbert space and $F(t)$ is a strongly continuous function from $(-\infty, \infty)$ to the unitary operators on $H$, such 
that strong $\lim _{|n| \rightarrow \infty} F(t / n)^{n}=U_{t}$ exists, then $U_{t}$ is a strongly continuous one-parameter unitary group.

REMARK. In 2.2 , assume that $R_{t}$ is a $\left(C_{0}\right)$ semigroup and let $A$ be its infinitesimal generator. From 2.1 we know that $A$ extends $F^{\prime}(0)$ if this operator is densely defined. It is worth noting that (at least under a certain technical auxiliary condition) $A$ extends $F^{\prime}(0)$ even if the latter is not densely defined.

3. Addition of unbounded operators. Let $U_{t}=e^{i t A}$ and $V_{t}=e^{i t B}$ be one-parameter unitary groups such that $\left(U_{t / n} V_{t / n}\right)^{n}$ converges in the strong topology to $W_{t}$. By 2.3 it follows that $W_{t}=e^{i t C}$ for some selfadjoint operator $C$. We regard $C$ as the sum of $A$ and $B$ in a generalized sense, and we write $C=A \oplus B$. We say that $A$ and $B$ are addable if the above limit exists. It is easy to see that $B \oplus A$ exists if $A \oplus B$ exists, and the two are equal. Thus this generalized addition is commutative. One also has $\lambda(A \oplus B)=\lambda A \oplus \lambda B$ for real scalars $\lambda$.

Unfortunately the operation $\oplus$ is not associative. Indeed, no reasonable associative addition process exists for unbounded selfadjoint operators, because $A, B \rightarrow \operatorname{cl}(A+B)$ is nonassociative. To see this, let $S$ be any densely defined symmetric operator with distinct selfadjoint extensions $A, B$. Then $\operatorname{cl}(A-B)=0$, i.e. $A \oplus(-B)=0$. Thus $(A \oplus(-B)) \oplus B=B$. But $A \oplus(-B \oplus B)=A$. Incidentally, this example also exhibits the failure of the cancellation law: we have $A \oplus(-B)=0=B \oplus(-B)$, though $A \neq B$. More drastic examples of nonassociativity exist; the common domain of $A \oplus(B \oplus C)$ and $(A \oplus B) \oplus C$ can reduce to $(0)$.

In the light of the above remarks the following result is reassuring; it is also interesting in that it shows that the addability of a pair of operators is stable under bounded perturbations.

Theorem 3.1. If $A \oplus B$ exists and $C$ is bounded then $A \oplus(B+C)$ exists and equals $A \oplus B+C$.

The proof makes use of a perturbation series; cf. [3, Theorem 13.2.1].

We also have the following result for positive operators.

Theorem 3.2. Suppose that $A$ and $B$ are positive and addable. Then $A \oplus B$ is positive.

4. Operators defined by Friedrichs extensions. Let $A$ and $B$ be positive selfadjoint operators on $H$. Let $H_{1}=D\left(A^{1 / 2}\right)$, a Hilbert space with respect to the "graph" norm $\|h\|_{1}=\left(\|h\|^{2}+\left\|A^{1 / 2} h\right\|^{2}\right)^{1 / 2}$. Then we have natural norm-decreasing inclusions $H_{1} \subset H \subset H_{1}^{*}$. Regard $B$, 
restricted to $H_{1}$, as a map from $H_{1}$ to $H_{1}^{*}$. If its closure $\hat{B}$ is selfadjoint (as an operator from a reflexive Banach space to its dual) we set $\hat{C}=\hat{A}+\hat{B}$. Let $C$ be the restriction of $\hat{C}$ to $D(C)=\{h \in D(\hat{C}): \hat{C} h \in H\}$. Then one can show that $C$ is a positive selfadjoint operator on $H$; it is an extension of $A+B$.

The following theorem generalizes a result of Faris [2].

THeOREM 4.1. Suppose either that $\hat{B}$ is bounded or that $\hat{B}$ is essentially selfadjoint on $D(A) \cap D(B)$. Then for $t \geqq 0$

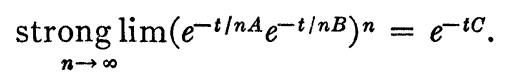

5. Boundedness of universally addable operators. If $A+B$ is densely defined we know by 2.1 that $A \oplus B$, if it exists, is a selfadjoint extension of the symmetric operator $A+B$. It is well known that symmetric operators exist which possess no selfadjoint extensions, and it is not hard to exhibit operators of the form $A+B$ with this property. However, much more is true.

THeOREM 5.1. Let $A$ be any unbounded selfadjoint operator. Then there exists a selfadjoint operator $B$ (necessarily unbounded) such that $A+B$ is a densely defined symmetric operator with deficiency indices $(1,0)$. In particular $A+B$ has no selfadjoint extensions.

It follows that if $A$ is unbounded $A \oplus B$ fails to exist for some $B$. Consequently, the only universally addable selfadjoint operators are the bounded ones.

\section{REFERENCES}

1. P. R. Chernoff, Note on product formulas for operator semigroups, J. Functional Analysis 2 (1968), 238-242. MR 37 \#6793.

2. W. G. Faris, The product formula for semigroups defined by Friedrichs extensions, Pacific J. Math. 22 (1967), 47-70. MR 35 \#5975.

3. E. Hille and R. S. Phillips, Functional analysis and semigroups, rev. ed., Amer. Math Soc. Colloq. Publ., vol. 31, Amer. Math. Soc., Providence, R. I., 1957. MR 19, 664.

4. E. Nelson, Feynman integrals and the Schrödinger equation, J. Math. Phys. 5 (1964), 332-343. MR 28 \#4397.

5. H. F. Trotter, On the product of semigroups of operators, Proc. Amer. Math. Soc. 10 (1959), 545-551. MR 21 \#7446.

University of California, Berkeley, California 94720 